\title{
Charles Perthées maps of palatinates: a project of digital edition
}

\author{
Tomasz Panecki ${ }^{\text {a, } * \text {, Tomasz Królik }}{ }^{\text {a }}$ \\ ${ }^{a}$ Tadeusz Manteuffel Institute of History of the Polish Academy of Sciences, tpanecki@ihpan.edu.pl, ta.krolik@student.uw.edu.pl \\ * Corresponding author
}

Keywords: digital edition, historical GIS, cartographic heritage, Poland, history

\begin{abstract}
:
Charles Perthées, who was a leading cartographer of King Stanisław August Poniatowski at the dawn of the PolishLithuanian Commonwealth, elaborated maps of palatinates (1:225,000, 1783-1804). They form a first map series of the Crown covering this area with the full settlement network, administrative borders, and natural environment. These maps were drawn with almost no field measurements or triangulation but on the basis of textual descriptions prepared by the parsons from 1778 to 1785 . The form of the maps is, however, similar to medium-scale topographic maps. Although the planimetric precision is much lower than on Austrian or Prussian maps of these times, Perthées' maps are much in detail (Fig. 1). For 21 years Perthées managed to elaborate 12 maps for 11 palatinates of the Crown after the First Partition. The maps serve as a primary source of information for settlement network reconstruction of the end of the 18th century and are extremely valuable for historians and geographers (Buczek 1966). The aim of the paper is to describe the idea behind their digital edition: digital representation of the maps and their content.
\end{abstract}

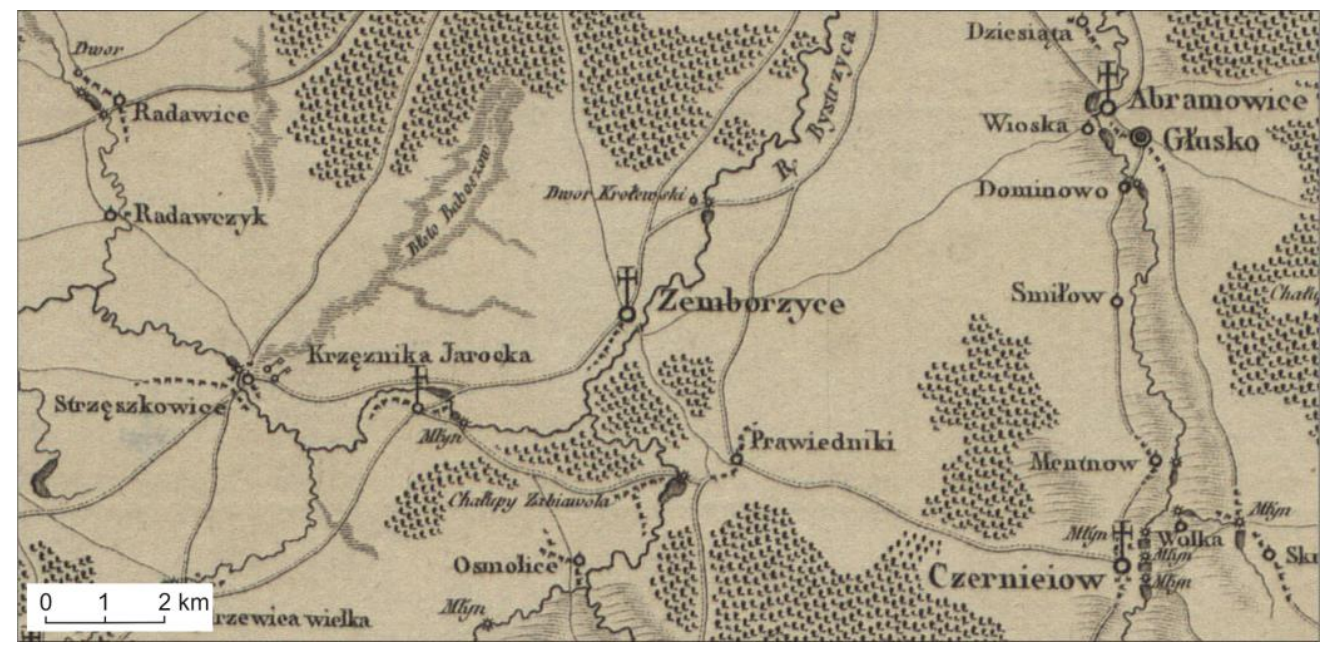

Figure 1. Fragment of the map covering Zemborzyce parish near Lublin (currently eastern Poland).

Editing of the maps has two objectives: (1) preparing maps and data derived from them for dissemination using FAIR principles including Linked Open Data (2) providing a set of information for the development of a historical map presenting settlements and boundaries of the Crown in the second half of the 18th century. The first objective requires that the map and its content should be represented digitally in the "source-driven" model: as close to the source as possible. The second entails that the data obtained from the maps should be georeferenced and related to the actual historical location of the features they represent. Combining these two objectives required a different approach to the vectorization of historic maps than the classic methodology based on map georeferencing and content vectorization.

The methodology, which we call the "source-driven approach", assumes treating maps as images with only a local reference system (XY Cartesian), and the data model is subordinated to the map legend. Therefore, the representation of their content is independent of a particular project. The approach involves three steps. In the first, the map sheets are entered into a GIS application and placed next to each other. Secondly, a database structure has been developed for maps' indexation consisting of five tables designed to represent the structure of the map content: "Maps", "Units", "Features", "Symbols" and "Annotations" (Fig. 2). Ultimately, map content is indexed (vectorized) and the whole process verified. 


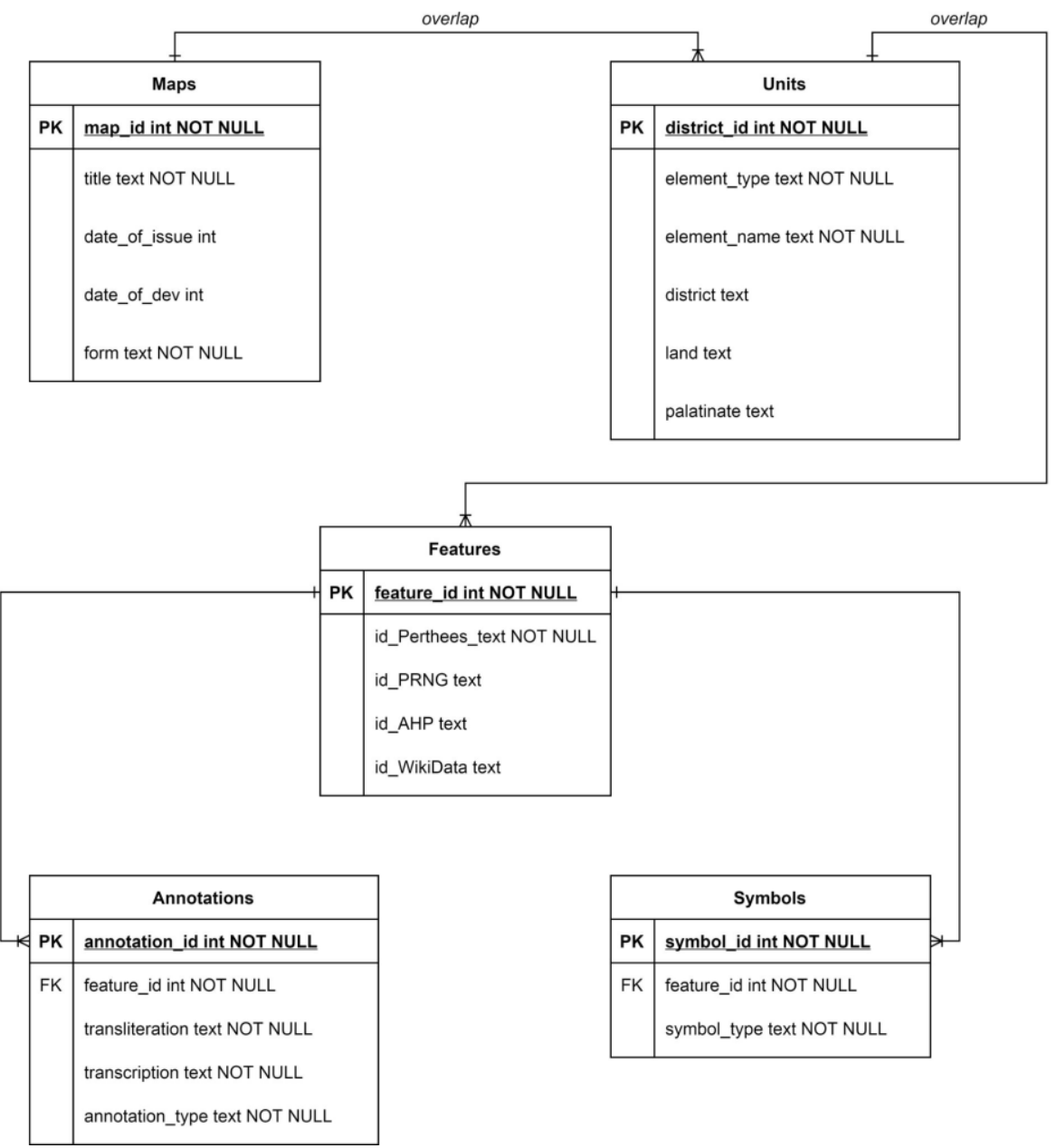

Figure 2. Database scheme for indexing the content of maps of palatinates.

The "Maps" table contains metadata with basic information about the particular map sheet that is indexed. Entities in this table are actually vector frames drawn around each of the sheets. In the "Units" table, data on homogeneous areas that can be distinguished on the map are collected. The areas can be of either geographical (district, palatinate) or graphic character (scale bar, legend, title, etc.). To maintain a "source-driven" model, it was decided to separate map symbols from map annotations and collect them in two different tables: "Symbols" and "Annotations" respectively. Preliminary studies have shown that it is often difficult to clearly link a symbol (e.g. village or mill) to its description (e.g. village name). Symbols and annotations, when indexed, have no relationship with each other. The polygons in the "Features" table, which spatially overlap with specific map symbols and annotations, allow relating them functionally and technically. At this stage, the features are preliminarily identified with external resources, like WikiData.

The result of such a procedure allows not only to present and share the map image and its data but also to use the acquired information for the elaboration of the historical maps of the Crown in the second half of the 18th century. Data prepared in the "source-driven" model enables reuse in other projects.

\section{Acknowledgments}

This research was funded by the Ministry of Science and Higher Education, Poland, grant number $11 \mathrm{H} 18012287$, "Cartography in the service of state reforms in the Stanisław epoch - a critical study of the "Geographic and statistical description of the parishes of the Kingdom of Poland" and maps of Karol Perthées' crown palatinates, 2019-2023”. 